\title{
Are cosmological $N$-body simulations reliable on scales below the mean separation length of particles?
}

\author{
Takashi Hamana \\ National Astronomical Observatory, Mitaka, Tokyo 181-8588, Japan
}

Naoki Yoshida

Harvard-Smithsonian Center for Astrophysics, 60 Garden Street, Cambridge, MA 02138, USA

\section{Yasushi Suto \\ Department of Physics, University of Tokyo, Tokyo 113-0033, Japan Research Center for the Early Universe (RESCEU), School of Science, University of Tokyo, Tokyo 113-0033, Japan}

\begin{abstract}
We critically examine the reliability of clustering below the mean separation length of particles in cosmological high-resolution $N$ body simulations. The particle discreteness effect imposes two fundamental limitations on those scales; the lack of the initial fluctuation power and the finite mass resolution. We address this problem applying the dark matter halo approach which makes us possible to examine separately how those two limitations affect the simulation results at later epochs. We find that the reliability of the simulations on scale below the mean particle separation is primarily determined by the mass of particles. Since the small scale matter clustering is dominated by the matter in a virialized halo, it is naturally expected that the small scale clustering becomes unreliable when the characteristic nonlinear mass for gravitationally collapsed objects at that time becomes smaller than the particle mass. This is confirmed by a detailed comparison with three major cosmological simulations. The characteristic mass, $M_{\mathrm{NL}}(z)$, is approximately given by the condition $\sigma\left(M_{\mathrm{NL}}, z\right)=1$. This mass scale should be much larger than the simulation particle mass $m_{\text {part }}$ so as to reproduce the proper amplitude of the small scale clustering. This requirement can be translated to the criterion for the critical redshift $z_{\text {crit }}$ when simulations reasonably resolve the clustering on scales below the mean separation: $M_{\mathrm{NL}}\left(z_{\text {crit }}\right)=n_{\text {halo }} m_{\text {part }}$, where we introduce a fudge factor, $n_{\text {halo }}$, of around ten. We conclude that at $z>z_{\text {crit }}$ high-resolution $N$-body simulations are not reliable on scales below the mean particle separation. Although it is not obvious if the opposite is true at $z<z_{\text {crit }}$, our detailed comparison suggests that it is indeed the case at least as far as the two-point correlation functions of the matter are concerned. The detail discussions are presented in Hamana, Yoshida \& Suto (2001).
\end{abstract}




\section{Discussion}

We examine the evolution of clustering in cosmological high-resolution $N$-body simulations taking explicit account of the two fundamental limitations: (i) the lack of the initial fluctuation power on scales smaller than the mean particle separation, and (ii) the finite mass resolution. To do this, we apply the dark matter halo approach in which the nonlinear dark matter two-point correlation function is well described by a sum of two contributions; one from the correlations between the halos (halo-halo correlation), and the other from correlations of matter within the same halo (the Poisson contribution).

Imposing the above two limitations on the dark matter halo approach, we found that the reliability of simulations below the mean separation length is really sensitive to those limitations. In particular, the effect is quite substantial at higher redshifts where the major fraction of mass is contained in less massive halos that cannot be resolved with a given particle mass in simulations. We compare the model predictions with three cosmological $N$-body simulations (Virgo SCDM \& $\Lambda$ CDM and Hubble volume $\Lambda$ CDM simulation) and found that the model well reproduces the defects in the simulations due to the above two limitations.

The model prediction together with the comparison with the $N$-body simulation clearly indicates that the small-scale clustering is primarily limited by the finite mass resolution. Also the Poisson term would be dominated by halos which become nonlinear at that epoch. The characteristic mass for gravitationally collapsed objects, $M_{\mathrm{NL}}(z)$, is approximately given by the condition $\sigma\left(M_{\mathrm{NL}}, z\right)=1$. Therefore this mass scale should be much larger than the simulation particle mass $m_{\text {part }}$ so as to reproduce the proper amplitude of the Poisson term. This requirement can be translated to the criterion for the critical redshift $z_{\text {crit }}$ when simulations reasonably resolve the clustering on scales below the mean separation: $M_{\mathrm{NL}}\left(z_{\text {crit }}\right)=n_{\text {halo }} m_{\text {part }}$, where we introduce a fudge factor $n_{\text {halo }}$ so as to represent the number of particles that is required to reasonably resolve a single halo of the nonlinear mass. In the previous simulations, $n_{\text {halo }}$ is typically supposed to be around 10. By a detailed comparison with three major cosmological simulations, we conclude that at $z>z_{\text {crit }}$ high-resolution $N$-body simulations are not reliable on scales below the mean particle separation.

Acknowledgments. T.H. thanks G.Börner and M.Bartlemann for the hospitality during his stay at MPA where most of the present work is performed. He also acknowledges support from Research Fellowships of the Japan Society for the Promotion of Science. The simulations used in this work were carried out by the Virgo Consortium and the data are publicly available at http://www.mpagarching.mpg.de/NumCos. This research was supported in part by the Grantin-Aid by the Ministry of Education, Science, Sports and Culture of Japan (07CE2002, 12640231).

\section{References}

Hamana, T., Yoshida, N., \& 2001, in preparation 